\title{
A CASE OF IMPENDING RUPTURE OF OVARIAN TUMOUR DURING THIRD TRIMESTER OF PREGNANCY
}

\author{
Lakshmikantha. G, Sheela. S. R, Chandra Mouli. A
}
1. Assistant Professor, Department of Obstetrics \& Gynaecology, Sri Devaraj Urs Medical College, Tamaka, Kolar, Karnataka, India.
2. Professor, Department of Obstetrics \& Gynaecology, Sri Devaraj Urs Medical College, Tamaka, Kolar, Karnataka, India.
3. Resident, Department of Obstetrics \& Gynaecology, Sri Devaraj Urs Medical College, Tamaka, Kolar, Karnataka, India.

\section{CORRESPONDING AUTHOR}

Dr. Lakshmikantha .G, \#249, $3^{\text {rd }}$ A main, Near Amba Bhavani circle, NE of NR Mohalla, Mysore, Karnataka, India

E-mail: drlakshmikanthag@yahoo.co.in

Ph: 00919740810611

CAPSULE: Pregnancy with undiagnosed huge ovarian cyst can still be a presenting feature in third trimester in developing countries.

KEY WORDS: Ovarian tumour, pregnancy

INTRODUCTION: Pregnancy with coexisting ovarian tumor is a rare association. Common types are cystic teratomas, paraovarian cysts, serous cystadenomas (1).These are usually diagnosed early in pregnancy due to booking visit and regular antenatal care. This helps in early treatment. We had a case who presented in late trimester with an impending rupture of an ovarian cyst as a consequence of pressure from the growing uterus.

CASE REPORT: A 25 yrs, Gravida 3 Para 2 Living 2 with previous normal deliveries with h/o 8 months of gestation, unbooked case, hailing from a rural village attended the emergency casualty in the early hours of June 2010 with h/o generalized abdominal pain for a few days which increased over last 2 hours. No h/o leaking or bleeding per vaginum. On examination she was moderately built and nourished with stable vitals. Per abdominal examination reveals generalised abdominal distension from pelvis up to xyphisternum with a demarcation line crossing obliquely from pubic symphysis up to left hypochondrium. Left to the demarcation line was a firm mass suggesting gravid uterus of 32 wks and right was a large tense cystic mass with tenderness. On Pelvic examination cervix was pushed to left, uneffaced, os closed, right and anterior fullness present.

Ultrasonography revealed a single live intra uterine pregnancy with gestational age 32 wks with large cystic lesion $22 \times 22 \times 20 \mathrm{cms}$ seen in right side of abdomen extending from pelvis up to right hypochondrium. No solid area noted in the cyst but minimal ascitis was present. The diagnosis of impending rupture of ovarian cyst with 32 wks pregnancy was made and proceeded with emergency laparotomy under prophylactic tocolytic cover with s/c terbutaline 250 micro grams. Intraoperatively an Uniloculated

30x20x20 cms right ovarian cyst with minimal Ascities was present. Fluid sent for cytology. About 6 litres of chocolate coloured fluid drained from the cyst before exteriorising it. 
Right tube was stretched over the cyst. Hence proceeded with right salphingo ovariotomy. Rest of the viscera appeared normal. Abdomen closed in layer with interrupted nonabsorbable stitches.

Post operatively prophylactic tocolytic and antibiotic administered. Uterus was relaxed and FHR was good. Advised for regular Antenatal checkups and patient discharged on day 10. Histology revealed it as serous cystadenoma ovary. She came for regular checkups and had spontaneous vaginal delivery of a live term male baby of weight $3.8 \mathrm{kgs}$ on 31-8-2010. post natal period uneventful.

DISCUSSION: The incidence of ovarian tumor complicating pregnancy is about 1 in 1000 . About $95 \%$ of ovarian tumors diagnosed during pregnancy are benign. Complications of cysts associated with pregnancy are torsion, rupture, infection, malignancy, malpresentation, obstructed labour (2). The diagnosis of coexisting tumor can be made early during antenatal care either by examination in early trimester or by ultrasonography and necessary treatment can be undertaken early(3).Usual treatment for large ovarian tumors during pregnancy is laparotomy during second trimester. However in developing countries large number of un booked cases come during late trimester, the complications are more common and the treatment becomes difficult(4). Our case is an example of unbooked status.

CONCLUSION: Providing health care facility, educating society regarding booking visit and regular antenatal care is the need of the hour to detect high risk cases early, especially in developing countries.

CONFLICT OF INTEREST: None to disclose.

\section{REFERENCES:}

1. 1.Lee CH, Raman S, Sivanesaratnam V: Torsion of ovarian tumors: a clinicopathological study: Int.J Gynaecol obstet 1989,28:21-25

2. Yen CF,Lin SL,Murk W et al:Risk analysis of torsion and malignancy for adnexal mass during pregnancy. Fertil Steril 2009,91(5):1895-902

3. Zaneth G,Mari ani E,Lissani A,et al. A Prospective study of the role of ultrasound in the management of adnexal masses in pregnancy. BJOG 2003; 110:578-583

4. Whitecar MP,Turners, Higby MK:Adnexal masses in pregnancy: A review of 130 cases undergoing surgical management. Am J Obstet gynecol 1999,181(1):19-24. 
FIGURE 1: Two separate masses showing firm mass below and cystic mass above.

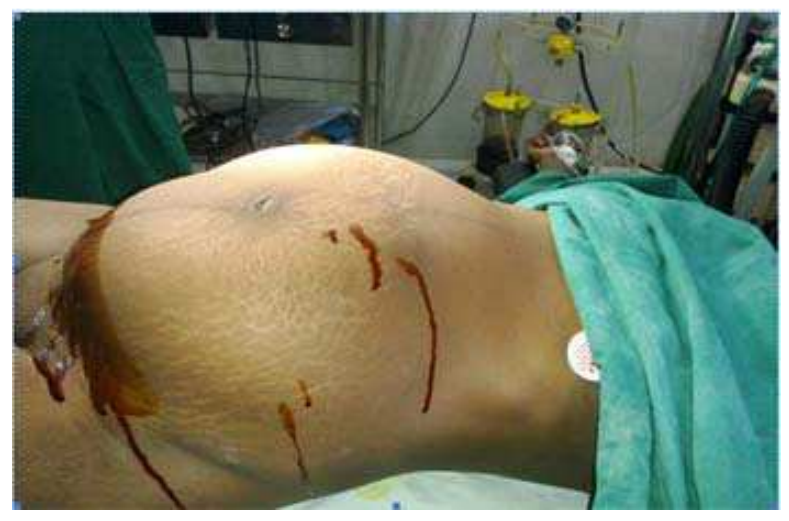

FIGURE 2: Intra operative finding showing ovarian cyst.

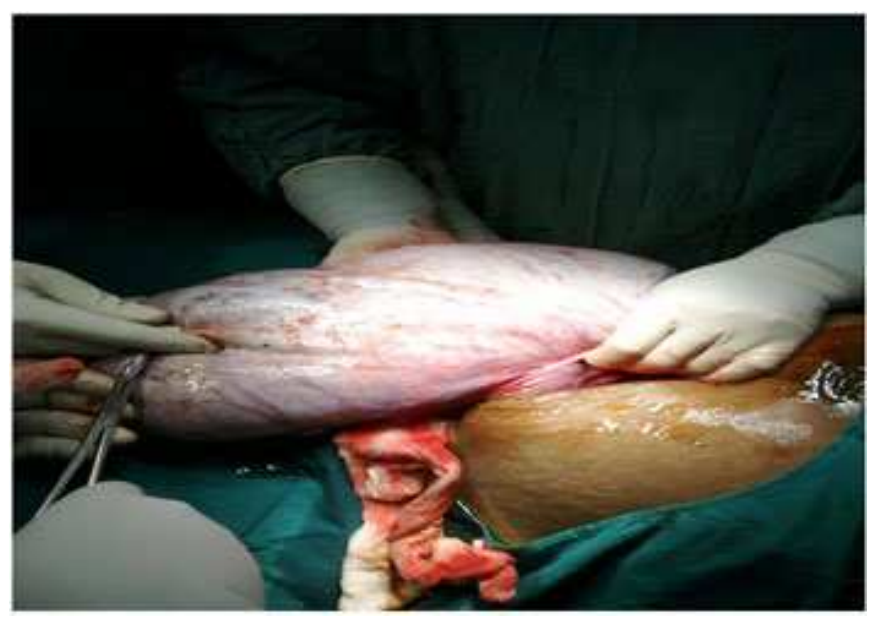

